\title{
Evolution of the pedestal on MAST and the implications for ELM power loadings
}

A. Kirk, G.F. Counsell, G. Cunningham, J. Dowling, M. Dunstan, H. Meyer, M. Price, S. Saarelma, R.Scannell 1), M. Walsh, H.R. Wilson 2) and the MAST team

EURATOM/UKAEA Fusion Association, Culham Science Centre, Abingdon, Oxon OX14 3DB, UK

1) Department of Electrical \& Electronic Engineering, University College Cork, Association EURATOM-DCU Ireland

2) University of York, Heslington, York YO10 5DD UK

e-mail contact of main author: andrew.kirk@ukaea.org.uk

\begin{abstract}
Studies of the pedestal characteristics and quantities determining ELM energy losses in MAST are presented. Progress is reported on the attempts to determine the quantities that affect the pedestal height and understanding ELM losses. High temperature pedestal plasmas have been achieved which have collisionalities one order of magnitude lower than previous results. The pedestal widths obtained in these low collisionality plasmas are in better agreement with banana orbit scalings than previous high collisionality plasmas, suggesting that banana orbits can only play a role in determining the minimum width when the collisionality is sufficiently low. A stability analysis performed on these plasmas shows them to be near the ballooning limit and to have broad mode structures which would predict large ELM energy losses. These ELM energy losses have been observed at the target resulting in peak power densities in excess of $\sim 20 \mathrm{MWm}^{-2}$. The fraction of pedestal energy released by an ELM as a function of collisionality has been compared with data from other devices. A model for ELM energy losses has been proposed and compared to data from MAST and JET.
\end{abstract}

\section{Introduction}

The high confinement mode of operation [1], or H-mode, is the standard operating regime envisaged for ITER [2]. The high confinement is achieved due to a narrow, insulating region, or transport barrier, that forms at the plasma edge as the heating power is increased above a threshold level. The pressure rises steeply from the plasma edge through the transport barrier, to create a "pedestal" on which the core pressure sits. The pedestal is located near the last closed magnetic flux surface and typically extends over a width of less than 5\%-10\% of the plasma minor radius. Some of the major outstanding plasma physics questions for fusion are associated with the properties of this narrow, but very important, region. In particular, high edge pressure gradients in the pedestal lead to a class of instabilities called Edge-Localised Modes, or ELMs [3][4]. These are violent events, which repetitively eject particles and energy from the plasma surface and may lead to unacceptably high-energy loads to the divertor plates and plasma facing components of future devices.

Extrapolations for the ELM size on ITER, based on data from existing tokamaks, indicate that the largest ELMs could not be tolerated regularly because of the damage they would cause. However, there is considerable uncertainty associated with such predictions because of the lack of a good scientific understanding of all of the processes involved. If we can improve our understanding of at least some aspects of the ELM physics, we may be able to constrain the models more, and hence reduce uncertainty in the predictions for ITER. Comparing results from various experiments [5] indicates that there is an ordering of the ELM energy loss with collisionality with increasing ELM size at low collisionality. On MAST the pedestal collisionality $\left(v_{p e d}^{*}\right)$ is typically large (1-3). In order to make the results more comparable to other devices and more relevant for extrapolation to 
ITER $\left(v_{p e d}^{*} \sim 0.03\right)$ it is important that discharges are produced and studied in MAST with lower pedestal collisionality i.e. higher temperature pedestal and/or lower density pedestal. In this paper new low collisionality discharges will be presented and the effect on ELM energy loss will be discussed. These observations will be combined with new results on the evolution of filamentary structures observed during ELMs in order to construct a model for ELM energy losses.

\section{Pedestal characteristics on MAST}

MAST has a major radius, $\mathrm{R} \sim 0.85 \mathrm{~m}$ and a minor radius, $\mathrm{a} \sim 0.65 \mathrm{~m}$. It has operated with plasma current up to $\mathrm{I}_{\mathrm{p}} \sim 1.35 \mathrm{MA}$ and is equipped with two neutral beam lines, which have so far provided deuterium injection into plasma with powers in excess of $\mathrm{P}_{\mathrm{NBI}} \sim 2.8 \mathrm{MW}$. The toroidal field on axis is usually in the range $0.45 \mathrm{~T}<\mathrm{B}_{\phi}<0.6 \mathrm{~T}$ but, due to the low aspect ratio, the toroidal field varies between around $1.2 \mathrm{~T}$ at the inboard mid-plane separatrix to $0.25 \mathrm{~T}$ at the outboard separatrix, this strong variation across the plasma being a distinct feature of the spherical tokamak.

Previous studies [6] of the pedestal parameters observed during ELMy H-mode periods in Connected Double Null (CDN) discharges on MAST have shown that the density pedestal $\left(\mathrm{n}_{\mathrm{e}}^{\text {ped }}\right.$ ) is in the range $2.0 \times 10^{19} \mathrm{~m}^{-3}$ to $7.1 \times 10^{19} \mathrm{~m}^{-3}$, the temperature pedestal $\left(\mathrm{T}_{\mathrm{e}}^{\text {ped }}\right)$ is between $\sim 80 \mathrm{eV}$ and $190 \mathrm{eV}$, the electron collisionality at the pedestal is in the range 1.0 to 5.0 and the pedestal stored energy is in the range 3 to $17 \mathrm{~kJ}$. The standard mode of operation uses fuelling from the High Field Side (HFS) midplane, as this has been found to reduce the power required for H-mode access [7]. The disadvantage of this technique is that due to the gas valve location, the gas flow cannot be

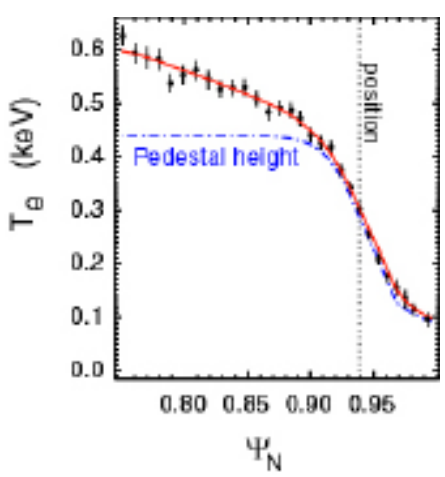

Figure 1 The temperature profile as a function of normalised flux. turned off during the shot and hence there is very little control over the neutral density in the vessel. Previous studies on MAST [6] have indicated that the neutral density and the neutral penetration length play a large role in determining the density pedestal. Therefore, with the aim of reducing the neutral density shots have been designed using optimized outboard gas fuelling and performed shortly after a boronisation of the vessel.

Figure 1 shows the temperature profile for the shot with the largest temperature pedestal observed to date on MAST. This shot has $\mathrm{n}^{\text {ped }}=(2.62 \pm 0.05) \times 10^{19} \mathrm{~m}^{-3}, \mathrm{~T}_{\mathrm{e}}^{\text {ped }}=435 \pm 20 \mathrm{eV}$, a

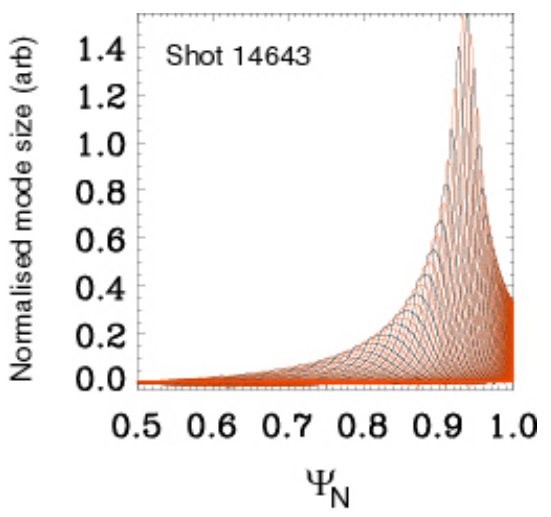

Figure 2 The radial mode structure of the most unstable mode. pressure pedestal $\left(\mathrm{p}_{\mathrm{e}}^{\mathrm{ped}}\right)=1.79 \pm 0.05 \mathrm{kPa}$ and a pedestal stored energy of $13.3 \mathrm{~kJ}$. This shot, which has a Single Null magnetic configuration, has pedestal collisionality $\mathrm{v}_{\text {ped }}^{*} \sim 0.08$. A comparison has been performed of a standard H-mode shot and one of these low collisionality shots. The pressure gradients are very similar in the two plasmas $\left(30.3 \mathrm{kPa} / \Psi_{\mathrm{N}}\right.$ for the high $\mathrm{v}_{\text {ped }}{ }^{*}$ case and $29.0 \mathrm{kPa} / \Psi_{\mathrm{N}}$ for the low $\mathrm{v}_{\text {ped }}^{*}$ case). However, the pressure pedestal width is more than twice as large in the low $\mathrm{V}_{p e d}^{*}$ case (high $\mathrm{V}_{p e d}^{*}: 0.017$ low $\mathrm{V}_{p e d}^{*}: 0.035 \Psi_{\mathrm{N}}$ ).

A stability analysis has been performed on the low 
$v_{p e d}^{*}$ shot where the profiles were obtained just before an ELM using the ELITE code [8]. The measured pressure gradient had to be increased by less than $10 \%$ in order to make the profiles unstable. The radial mode structure of the most unstable mode $(n=20)$ is shown in figure 2. As can be seen, the mode extends deep into the core. A stability diagram has been produced (figure 3 ) which shows that the experimental point lies close to the pressure boundary a trait often associated with type I ELMs.

Previous studies have shown that the temperature pedestal widths on MAST were lower than the banana orbit scaling and it was speculated at that time that this may be due to the fact that they were at high collisionality [6]. Having now achieved a lower collisionality pedestal it is interesting to compare these new discharges with the scaling (figure 4) from other devices [9]. Although most of the new points still lie below the line they are closer to it and do show some correlation. In fact the lowest collisionality point lies on the line. This may well indicate that the banana orbit effects are playing a role in setting the minimum pedestal width but that these effects are removed at high collisionality.

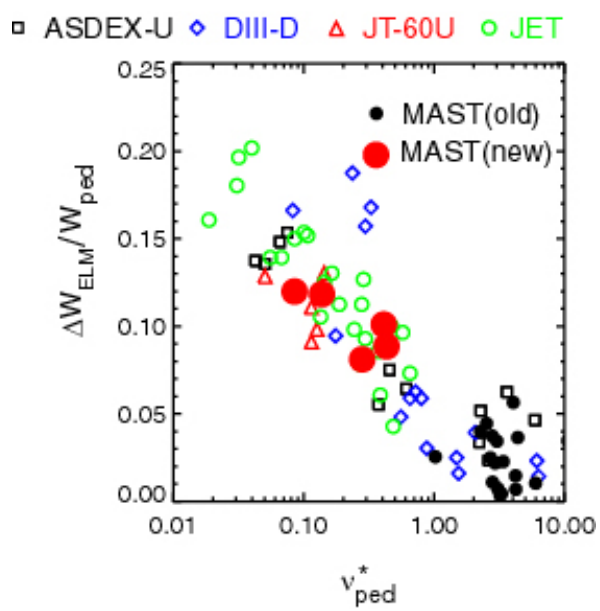

Figure 5 ELM energy loss as a fraction of pedestal energy versus pedestal collisionality for data from MAST and other devices [5].

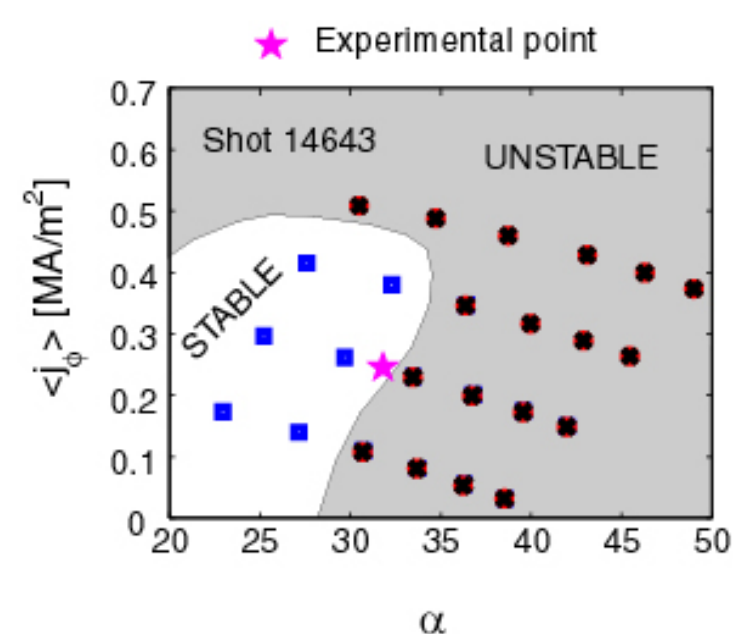

Figure 3 Stability diagram plots from ELITE showing edge current density versus normalised pressure gradient.

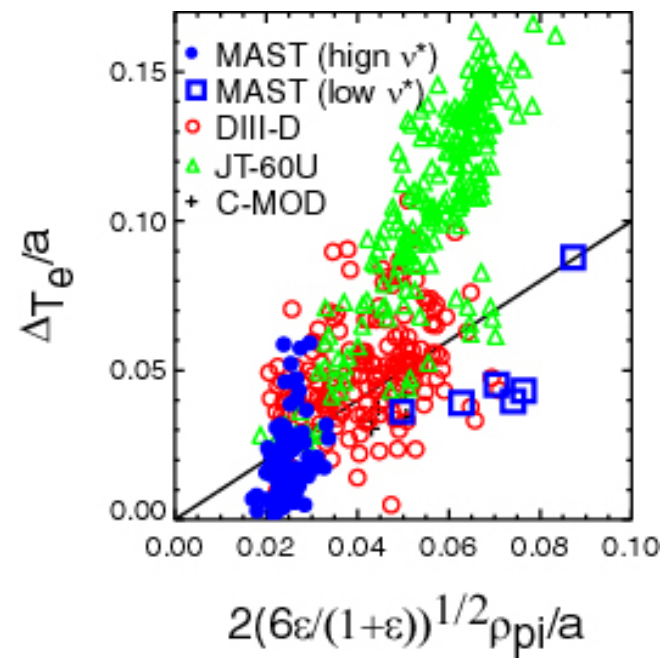

Figure 4 A comparison of MAST data with data from other tokamaks [9] for the normalised temperature pedestal versus the banana orbit width normalised to the minor radius.

\section{The evolution of the pedestal and energy losses during ELMs}

Figure 5 shows a plot of the fraction of the pedestal energy released by an $\operatorname{ELM}\left(\Delta \mathrm{W}_{\mathrm{ELM}} / \mathrm{W}_{\mathrm{ped}}\right)$ as a function of pedestal collisionality for data [5] from JET, DIII-D, JT-60 U and ASDEX Upgrade to which data from MAST has been added. As has been observed on other devices the fraction of the pedestal energy released by a Type I ELM is found to increase as edge collisionality decreases. At high collisionality on MAST 
the fraction of pedestal energy released is in the range 1-5\%, i.e. a maximum of $500 \mathrm{~J}$ released by the ELM. In the low collisionality cases this rise to $13-15 \%\left(\Delta \mathrm{W}_{\mathrm{ELM}} \sim 1.3-1.7 \mathrm{~kJ}\right)$ and the peak power loads to the outboard divertor targets rises from $\sim 2-4 \mathrm{MWm}^{-2}$ to $24 \mathrm{MWm}^{-2}$.

The time evolution of the pedestal can be determined using the new multi-time point edge Nd-YAG Thomson scattering system (TS) on MAST. It consists of 4 lasers which are aligned radially at the mid-plane. The spatial resolution in the edge region is $\sim 1 \mathrm{~cm}$ and each Nd-YAG laser can fire once every $20 \mathrm{~ms}$. The relative timing of the lasers can be adjusted such that electron density and temperature radial profiles can be produced with as little as a $1 \mu$ s delay between them. Additional information on the evolution of the total plasma density comes from an interferometer system that measures the line integral density on a similar radial path through the plasma and which can be digitized at up to $1 \mathrm{MHz}$. During an ELM on MAST and all other devices, the fractional change in density pedestal is effectively independent of the precrash pedestal conditions with $\sim 3.4 \%$ of the preELM particles being expelled by the ELM. Figure 6 shows the evolution of the pedestal temperature and density profiles (separated by $200 \mu \mathrm{s}$ ) together with the line average density for a typical high collisionality ELM. The profiles obtained just before the ELM are shown in blue. The subsequent profiles show that the pedestal density drops by $\sim 25 \%$ by the time of the peak of the midplane $\mathrm{D}_{\alpha}$ signal (red profiles) and does not drop any further after that. Hence the losses from the core have occurred during the rise time of the $\mathrm{D}_{\alpha}$

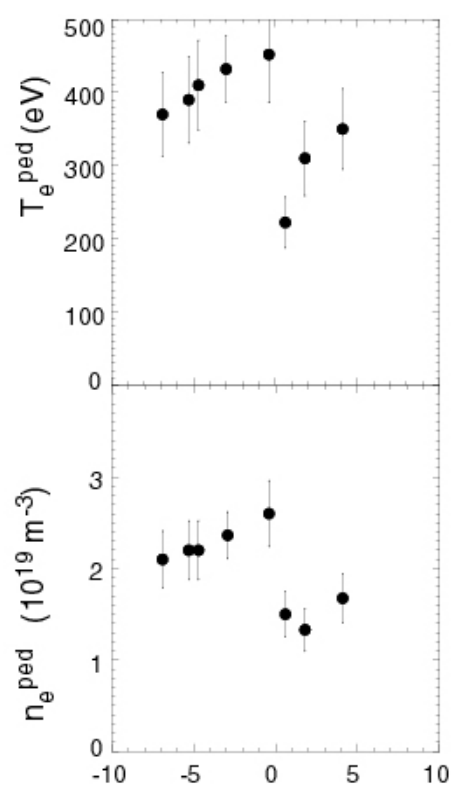

Time relative to ELM (ms)

Figure 7 Evolution of the temperature and density pedestal with respect to the nearest ELM in a low collisionality shot. signal. The line integral density measurement drops on a longer time scale due to the fact that it is measuring not only the core but also the scrape off layer (SOL). The density in the SOL has been increased due to the ELM and
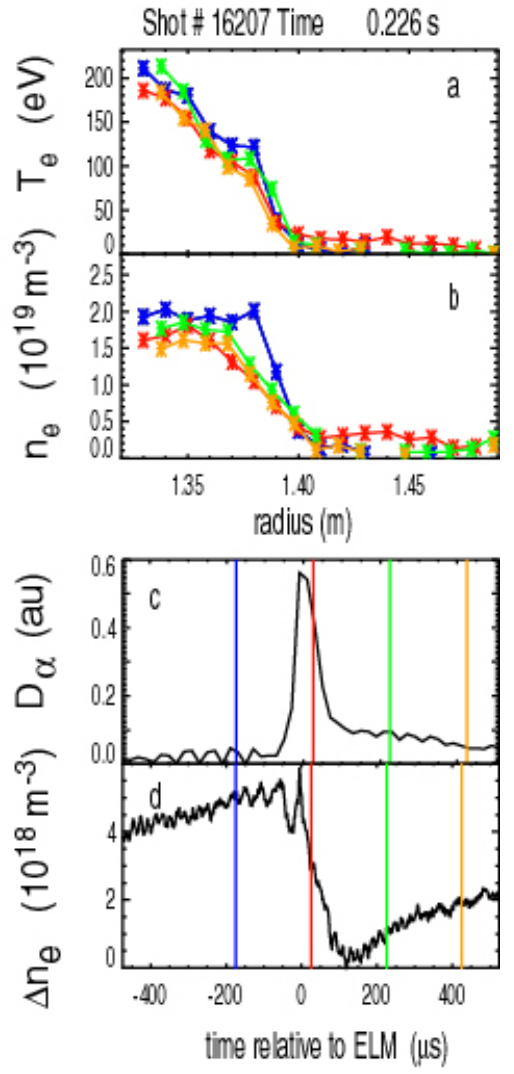

Figure 6 a) the temperature and $b$ ) the density profiles obtained at times relative to $c$ ) the midplane $D_{\alpha}$ intensity and $d)$ the change in the line averaged density. it is depleted on the ion parallel transit time. The temperature profiles show that there is effectively no change to the temperature pedestal due to ELMs with a high collisionality pedestal.

The ELM frequency in the low collisionality case is much lower and the probability of recording a set of (non-triggered) profiles through one of these ELMs is small. Therefore, in this case the TS lasers were evenly spaced and a profile recorded every $5 \mathrm{~ms}$. By combining the results from 2 similar shots the pedestal evolution during the ELM cycle of a low collisionality regime can be 
produced (see figure 7). In this case there is a substantial reduction in the temperature pedestal.

\section{Filaments during ELMs}

Scalings, such as those observed in figure 5, have been used to predict an ELM energy loss on ITER [5]. Rather than relying on scalings, however, it would be better to construct a model for the ELM energy loss process itself. Filament structures have been observed during ELMs in a wide range of Tokamaks using a variety of diagnostics (see [10] and reference therein). Although it is clear that these structures exist during the ELM it has been unclear as to what role they play in the energy loss process. Measurements have been performed on MAST in order to resolve these uncertainties by studying the 3-D propagation of the filaments as a function of time during the ELM for a range of pedestal collisionalites. The unique wide angle view of the plasma and the large gap $(>50 \mathrm{~cm}$ at the outboard mid-plane) between the Last Closed Flux Surface (LCFS) and the vessel makes MAST the ideal machine on which to perform such studies as it allows the radial propagation of the filaments to be tracked without interactions with vessel components.
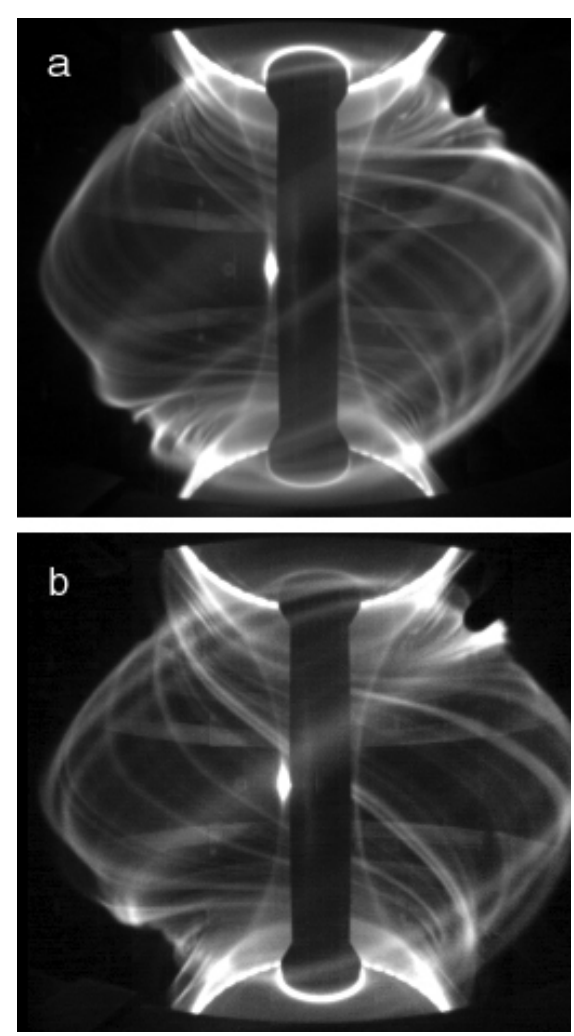

Figure 8 Visible images obtained during an ELM for a MAST plasma with a) $v^{*}=0.5$ and $b$ ) $v *=4$

The images described in this paper come from a Photron Ultima APX-RS camera, which can be used to capture a continuous set of images throughout the entire shot. In this present analysis it has been used with a $5 \mu$ s exposure time in two modes: either a full plasma view (512x462 pixels) at $7.5 \mathrm{kHz}$ framing rate or a view of a region of the edge (256x48 pixels) at $100 \mathrm{kHz}$. Figure 8 a and $\mathrm{b}$ shows typical visible light images obtained using a $5 \mu$ s exposure during the rise time of the midplane $D_{\alpha}$ signal for two ELMs from discharges with different pedestal collisionalities $\left(v_{\text {ped }}^{*}=0.55\right.$ and 4.0 respectively). The ELM energy loss $\left(\Delta \mathrm{W}_{\mathrm{ELM}}\right)$ in the two cases is 1.4 and $0.3 \mathrm{~kJ}$ respectively. Clear filamentary structures are observed for both ELMs. The images have been analysed by mapping 3-D field lines, generated from the magnetic equilibrium at various distances outside the LCFS, onto the 2-D image [11]. The toroidal angle of these projected field lines is then modified so as to minimise the difference between the mapped field line and the observed filament. The filaments observed during ELMs appear to be aligned with the local field line.

In Figure 8a 12 filaments are observed at toroidal locations of 5, 29, 59, 103, 135, 168, $215,241,282,302,322$ and 350 degrees. Firstly it can be noted that the filaments are not equally spaced and only a quasi-mode number can be extracted. As will be shown below this is because the angular velocity of the filaments changes during the ELM and different filaments slow down at different times. Secondly, the radial location of the filaments with respect to the LCFS $\left(\Delta \mathrm{R}_{\mathrm{LCFS}}\right)$ is zero for all the filaments except for the ones at 5, 29 and 350 degrees, which are at $\Delta \mathrm{R}_{\mathrm{LCFS}}=2,6$ and $4 \mathrm{~cm}$ respectively. This indicates that filaments leave the LCFS at different times during the ELM. Thirdly, the width of the filaments varies between 5 and $10 \mathrm{~cm}$. Since the light is being emitted from a shell around the edge of the filament, those located at the far side of the plasma look split i.e. there looks like there are two filaments which are separated by the angular width of those 
observed at the near side of the plasma. In Figure 8b 12 filaments can be observed at 20, 73, 108, $144,167,197,225,263,295,318$, and 335 degrees. All are compatible with being on the LCFS except those at 41,73 and 318 degrees which have $\Delta R_{\mathrm{LCFS}}=4,2$ and $3 \mathrm{~cm}$ respectively. The filament widths again vary between 5 and $10 \mathrm{~cm}$. Hence, it does not seem to be the size or the

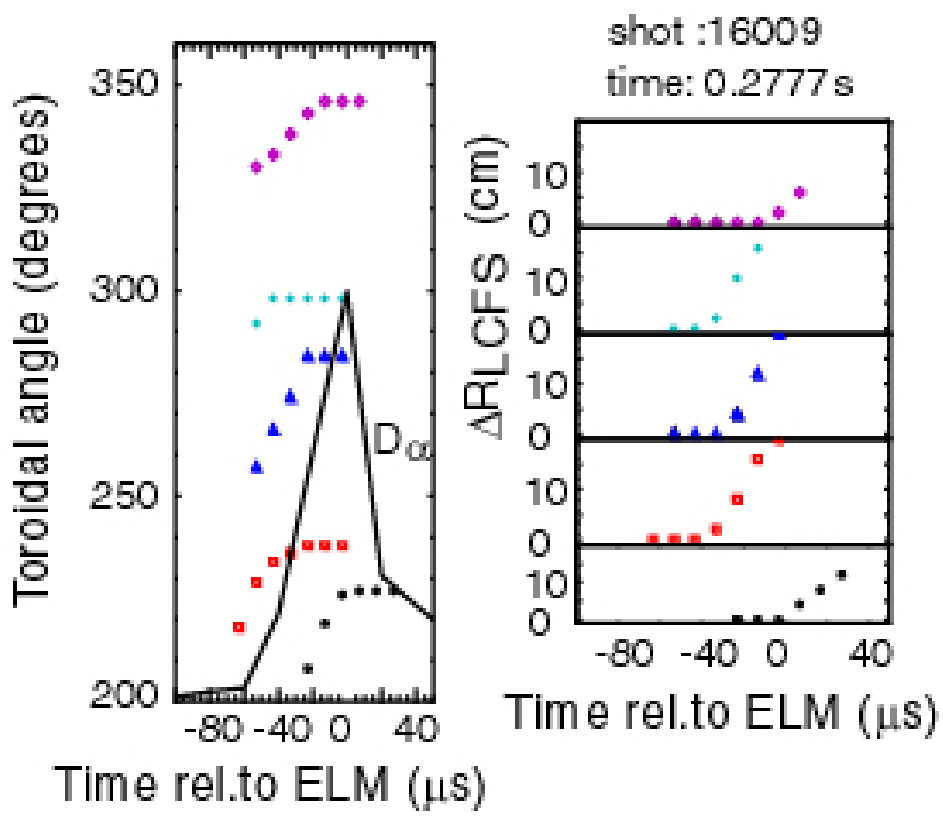

Figure 9 The toroidal angle and radial distance outside the LCFS $\left(\triangle R_{L C F S}\right)$ as a function of time during the ELM. number of filaments that are responsible for the large difference in ELM energy loss as a function of collisionality.

The propagation of the filaments observed during ELMs has been determined by measuring their toroidal and radial location in subsequent frames, separated by $10 \mu \mathrm{s}$, using a reduced window frame, centred on the midplane. Figure 9 shows the evolution of 5 filaments that have been tracked during a single ELM. All the filaments start off rotating toroidally in the co-current direction at 20 $\mathrm{kms}^{-1}$, which is similar to the rotation speed of the pedestal region. The filaments remain on the

LCFS for at least $\sim 50 \mu \mathrm{s}$; sometime after this the toroidal rotation of an individual filament slows and as it does so it accelerates radially outwards. The toroidal deceleration is in the range of (1.5$3.0) \times 10^{8} \mathrm{~ms}^{-2}$ and the radial acceleration in the range $(1.0-2.5) \times 10^{8} \mathrm{~ms}^{-2}$. Filaments are observed to persist through the rise time of the midplane $D_{\alpha}$, which as discussed above (see figure 6 ) is the time during which particles are lost from the pedestal.

Further information on the nature of the filaments comes from a reciprocating probe which is equipped with Langmuir probes and set of magnetic coils. A magnetic signal is detected which is correlated with the peaks in the ion saturation current i.e. with the arrival of the filament at the probe. The spectrogram of the magnetic signal shows two components: a high frequency component centred at $\sim 380 \mathrm{kHz}$, which is similar to the Alfvén frequency $(300-600 \mathrm{kHz})$ for the densities and temperatures of the filament, and a low frequency component. This low frequency component is consistent with the filament carrying a current of $\sim 190$ A that is aligned with the local B field and travelling in a mainly radial direction. This is consistent with the direction of propagation observed in the visible images. It is interesting to note that the current in the filament is similar to the calculated edge bootstrap current density for this shot $\left(\sim 0.1 \mathrm{MAm}^{-2}\right)$ multiplied by the cross section area of the filament $\left(2 \times 10^{-3} \mathrm{~m}^{2}\right)$ which is $200 \mathrm{~A}$.

The edge TS system has been used to obtain density and temperature profiles, separated by $5 \mu \mathrm{s}$, during the rise time of the ELM. These have been used to determine the particle and energy content of the filaments [11]. It has been found that at the time the filaments leave the LCFS each one contains at most $2.5 \%$ of the number of particles and $1.5 \%$ of the energy expelled by the ELM. In fact, the total loss from 10 similarly sized filaments (and usually they are smaller) would only represent $25 \%$ of the particles and $15 \%$ of the energy lost from the core due to the ELM. 


\section{A simple model for ELM energy losses}

The results presented in this paper show that filaments exist during the time that the losses occur from the pedestal. Based on these observations the most probable ELM energy loss process is as follows: for the first 50-100 $\mu$ s filaments remain near to the LCFS. During this time the filaments enhance losses from the pedestal region either because they have perturbed the field lines and increased cross field transport or because they provide a direct path from the pedestal region into the SOL. After this time the filaments accelerate radially away from the LCFS. Since their measured density and temperature decrease with distance it suggests that a reconnection event has occurred and that the filaments no longer remain attached to the core. At the time of the reconnection 50$75 \%$ of the total ELM particle $\left(\Delta \mathrm{N}_{\mathrm{ELM}}\right)$ and energy $\left(\Delta \mathrm{W}_{\mathrm{ELM}}\right)$ loss from the core has already occurred and each filament contains up to $2.5 \%$ of $\Delta \mathrm{N}_{\mathrm{ELM}}$ and $\Delta \mathrm{W}_{\mathrm{ELM}}$ which is subsequently lost by parallel transport along open field lines to the targets.

Based on these observations it is possible to construct a simple model for energy and particle losses during an ELM, in which it is assumed that the filament structures are linked to the core during a time $\tau_{\mathrm{ELM}}$. During this time the filament acts as a conduit for losses from the pedestal region into the SOL either by convective parallel transport due to a reconnection process or by increasing the cross-field transport into the SOL. According to the predictions of non-linear ballooning mode theory, close to marginal stability $\tau_{\mathrm{ELM}} \sim\left(\tau_{\mathrm{A}}{ }^{2} \tau_{\mathrm{E}}\right)^{1 / 3}$, where $\tau_{\mathrm{A}}$ is the Alfvén time and $\tau_{\mathrm{E}}$ is the energy confinement time. The total number of particles that could flow down $n$ filaments is given by $N_{f i l}=n \Gamma \sigma_{f i l} \tau_{E L M}$, where $\Gamma$ is the average particle flux density and $\sigma_{f i l}$ is the cross sectional area of the filament. The average particle flux density is calculated using the pedestal density and temperature respectively. The energy lost due to an ELM would then be $\Delta W_{E L M}=\frac{3}{2} k\left(T_{i}^{p e d}+T_{e}^{p e d}\right) N_{\text {fil }}$. Assuming the filaments have a circular cross section and using the measured widths the ELM energy losses obtained from the model (assuming $T_{i}{ }^{\text {ped }}=T_{e}^{\text {ped }}$ ) compared to the measured energy losses on MAST are shown in figure 10. Although slightly underestimating the ELM energy loss the model predictions are in reasonable agreement with the data. In fact since this model only tries to predict the losses up to the time that the filament detaches it might be expected that it would only predict $\sim 75 \%$ of the total losses. In order to see if such a model is valid for other devices, published ELM energy loss data from JET [12] has been used. It has been assumed that the filament size scales as $\sigma_{f i l} \propto$ (minor radius $)^{2}$ and that there are 10 filaments present. The model gives a reasonable agreement with the data, if anything underestimating the ELM energy loses. Although this model is very simple it highlights some of the things we do not know, for example how does the filament size scale with device size and does it have a circular cross section? The scaling used here gives a circular filament with a diameter of $20 \mathrm{~cm}$ on JET. This is larger than the gap between the plasma and the limiter. In order to avoid a direct path from the core to the limiter and hence large first wall power loadings, which are not observed, the majority of ELM particle

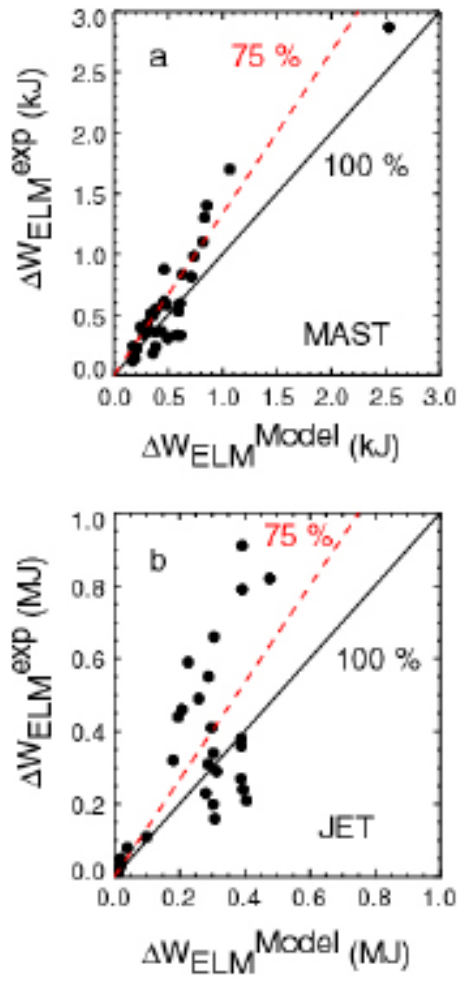

Figure 10 A plot of the measured ELM energy loss versus the ELM energy loss calculated from the data a) MAST and b) JET. 
and energy loss would have to occur while the largest part of the filament was still inside the LCFS. This would imply that when the filament detaches it would leave a localised density hole behind inside the LCFS. This may be a possible process since evidence for such holes has been reported on MAST [11] and ASDEX Upgrade [13]. It is possible that such a free streaming model could also explain why at low collisionalities there is a reduction in the temperature pedestal while at high collisionalities there is no reduction. At low collisionalities the ion thermalisation time $\left(\tau_{\mathrm{th}}\right)$ is long compared to $\tau_{\mathrm{ELM}}$ (e.g. $\tau_{\mathrm{th}}=900 \mu \mathrm{s}$ and $\tau_{\mathrm{ELM}}=100 \mu \mathrm{s}$ for the lowest collisionality case in MAST). During this time the fastest particles will be lost from the pedestal region leading to a shift in the mean of the Maxwellian velocity distribution to a lower value and hence a measured temperature drop. Conversely at high collisionality $\tau_{\text {th }} \leq \tau_{\mathrm{ELM}}$ (e.g. for a high collisionality discharge on MAST $\tau_{\text {th }} \sim 50$ $\mu \mathrm{s})$ and the particles in the pedestal region thermalise during the process, and hence the velocity distribution is affected evenly, resulting in effectively no measured temperature drop. Although the numbers appear to work on MAST they need to be checked on other devices.

\section{Summary}

High temperature pedestal plasmas have been achieved on MAST with collisionalities one order of magnitude lower than in previous discharges. A stability analysis performed on these plasmas shows them to be near the ballooning limit and to have broad mode structures which would predict large ELM energy losses. These losses have been observed at the target resulting in peak power densities in excess of $20 \mathrm{MWm}^{-2}$. The fraction of pedestal energy released by an ELM as a function of collisionality on MAST is found to be in agreement with data from other devices. The evolution of the filamentary structures observed during ELMs has been studied at both high and low collisionality and a simple model has been developed to predict ELM energy losses. One of the aims of such a simple model is to try to highlight questions that need to be answered in future experiments.

\section{Acknowledgements}

UKAEA authors were funded jointly by the United Kingdom Engineering and Physical Sciences Research Council and by the European Communities under the contract of Association between EURATOM and UKAEA. The views and opinions expressed herein do not necessarily reflect those of the European Commission.

\section{References}

[1] F. Wagner et al., Phys. Rev. Lett. 49, 1408 (1982).

[2] ITER Physics Expert Group, Nucl. Fusion 39, 2391 (1999).

[3] J. W. Connor, Plasma Phys. Control. Fusion 40, 531 (1998).

[4] W. Suttrop, Plasma Phys. Control. Fusion 42, A1 (2000).

[5] A. Loarte et al., J. Nucl. Mater. 313-316 (2003) 962.

[6] A. Kirk et al., Plasma Phys. Control. Fusion 46, 551 (2004).

[7] A.R. Field et al., Plasma Phys. and Control. Fusion 46 (2004) 981.

[8] H.R. Wilson et al., Physics of Plasmas 9 (2002) 1277.

[9] T.H. Osborne et al., IAEA-CN-94 /CT-3, IAEA Fusion Energy Conf., Lyon, France, 2002.

[10] A. Kirk et al., Plasma Phys. Control. Fusion 47, 995 (2005).

[11] A. Kirk et al., Phys. Rev. Lett. 96, 185001 (2006).

[12] D. Testa et al., Plasma Phys. Control. Fusion 47, 733 (2005).

[13] B. Kurzan et al., Phys. Rev. Lett. 95, 145001 (2005). 